\title{
A two-layered machine learning method to identify protein O-GlcNAcylation sites with O- GlcNAc transferase substrate motifs
}

\author{
Hui-Ju Kao ${ }^{1 \dagger}$, Chien-Hsun Huang ${ }^{1,2+}{ }^{+}$, Neil Arvin Bretaña ${ }^{3}$, Cheng-Tsung Lu', Kai-Yao Huang ${ }^{1}$, Shun-Long Weng ${ }^{4,5,6^{*}}$, \\ Tzong-Yi Lee L,7* $^{1,}$
}

From Joint 26th Genome Informatics Workshop and Asia Pacific Bioinformatics Network (APBioNet) 14th International Conference on Bioinformatics (GIW/InCoB2015)

Tokyo, Japan. 9-11 September 2015

\begin{abstract}
Protein O-GlcNAcylation, involving the $\beta$-attachment of single $N$-acetylglucosamine (GlcNAc) to the hydroxyl group of serine or threonine residues, is an O-linked glycosylation catalyzed by O-GlcNAc transferase (OGT). Molecular level investigation of the basis for OGT's substrate specificity should aid understanding how O-GlcNAc contributes to diverse cellular processes. Due to an increasing number of O-GlcNAcylated peptides with site-specific information identified by mass spectrometry (MS)-based proteomics, we were motivated to characterize substrate site motifs of O-GlcNAc transferases. In this investigation, a non-redundant dataset of 410 experimentally verified O-GlcNAcylation sites were manually extracted from dbOGAP, OGlycBase and UniProtKB. After detection of conserved motifs by using maximal dependence decomposition, profile hidden Markov model (profile HMM) was adopted to learn a first-layered model for each identified OGT substrate motif. Support Vector Machine (SVM) was then used to generate a second-layered model learned from the output values of profile HMMs in first layer. The two-layered predictive model was evaluated using a five-fold cross validation which yielded a sensitivity of $85.4 \%$, a specificity of $84.1 \%$, and an accuracy of $84.7 \%$. Additionally, an independent testing set from PhosphositePlus, which was really non-homologous to the training data of predictive model, was used to demonstrate that the proposed method could provide a promising accuracy (84.05\%) and outperform other O-GlcNAcylation site prediction tools. A case study indicated that the proposed method could be a feasible means of conducting preliminary analyses of protein O-GlcNAcylation and has been implemented as a web-based system, OGTSite, which is now freely available at http://csb.cse.yzu.edu.tw/OGTSite/.
\end{abstract}

\section{Introduction}

A type of O-linked glycosylation, Protein O-GlcNAcylation (O-GlcNAc), attaches a single $\mathrm{N}$-acetylglucosamine (GlcNAc) to serine (Ser)/threonine (Thr) residues [1]. O-GlcNAc, commonly found on cytoplasmic and nuclear proteins, has been shown to modulate molecular processes

\footnotetext{
*Correspondence: 4467@mmh.org.tw; francis@saturn.yzu.edu.tw

+ Contributed equally

'Department of Computer Science and Engineering, Yuan Ze University,

Taoyuan 320, Taiwan

${ }^{4}$ Department of Obstetrics and Gynecology, Hsinchu Mackay Memorial

Hospital, Hsin-Chu 300, Taiwan

Full list of author information is available at the end of the article
}

and cellular processes [2]. O-GlcNAc transferase (OGT) is an enzyme responsible for the addition of O-GlcNAc during glycosylation. On the other hand, an enzyme O-GlcNAcase (OGA) can remove O-GlcNAc. Recently, extracellular O-linked $\beta$-N-acetylglucosamine (EOGT) [3], an atypical OGT, has been reported to be responsible for extracellular O-GlcNAcylation of secreted and membrane glycoproteins [4]. Protein O-GlcNAcylation is also responsible for regulating cell-cell and cell-matrix interactions [5]. Accumulating evidence suggests that OGTs may act as a nutrient sensor that links hexosamine biosynthesis pathway to oncogenic signaling and regulation of factors involved in glucose and lipid metabolism [6]. 
The O-GlcNAc-dependent regulation seems to play an important role in the signaling pathways involved in metabolic reprograming of cancer cells [7]. In addition, O-GlcNAcylation is also an important post-translational modification and deregulation of this mechanism has been linked to various diseases such as diabetes [8], Alzheimer disease [9] and cancers [10-12].

With the improvement in mass spectrometry technologies, O-GlcNAcylated proteins in postsynaptic density [13], murine synapse [14], mouse brain [15], rat brain [16], mouse embryonic stem cell [17], and Hela cells [18], have been identified in recent years. However, precise identification of O-GlcNAcylation sites remains to be a challenge due to its dynamic characteristics [19]. Due to an interest to better identify O-GlcNAcylation sites and reduce experimental efforts, computational prediction of site motifs and O-GlcNAcylation sites have been considered. Previously, Gupta and Brunak have developed YinOYang an O-GlcNAcylation prediction tool trained using 40 O-GlcNAcylation sites [20]. Chen et al. have developed a similar tool incorporating structural topology to identify $\mathrm{O}$-glycosylation sites on transmembrane proteins [21]. The increase in experimentally identified O-GlcNAcylation sites motivates new developments including OGlcNAcScan, which was trained using 373 O-GlcNAcylation sites [22]. More recently, a new prediction tool, O-GlcNAcPRED, has been proposed claiming to have better performance than the aforementioned tools [23]. In the midst of these developments, Carage et al. have demonstrated that ensembles of support vector machine (SVM) classifiers could outperform single SVM classifier in terms of predicting protein glycosylation sites [24].

Although several computational methods have been developed to predict protein O-GlcNAcylation sites, there is currently no such tool that includes the investigation of potential OGT substrate motifs. It has been reported that molecular level investigation on OGT substrate specificity may aid in understanding how O-GlcNAc contributes to a diverse set of cellular processes [25]. With this, we were motivated to characterize O-GlcNAcylation sites with the consideration of amino acid composition [26]. In this study, we apply maximal dependence decomposition (MDD) to explore potential OGT substrate motifs for the experimentally verified O-GlcNAcylation sites. Statistically significant substrate motifs were further tested its prediction power by cross-validation evaluation and independent testing. A two-layered machine learning method, incorporating profile hidden Markov model (HMM) and support vector machine (SVM), was utilized to construct the predictive models. Furthermore, to facilitate the study of protein O-GlcNAcylation, MDD-identified substrate motifs were exploited to implement a web-based tool for identifying O-GlcNAcylation sites with corresponding OGT substrate motifs.

\section{Material and methods}

\section{Construction of positive and negative training data sets}

Due to the high-throughput mass spectrometry-based glycol-proteomics [27], several databases [22,28-30] have been developed for cumulating experimentally verified O-GlcNAcylation sites by manually surveying the glycosylation-associated literatures. In this work, the data set for training the predictive model of O-GlcNAcylation sites was mainly extracted from dbOGAP [22], O-GlycBase [31], and UniProtKB [32]. From dbOGAP, a total of 250 and 142 sites for O-GlcNAcylated serine (Ser) and threonine (Thr) on 172 proteins were collected. From O-GlycBase version 6.0, 24 sites for O-GlcNAcylated Ser and Thr from 17 proteins were collected. In UniProtKB, experimentally verified O-GlcNAcylation data were first filtered by removing entries annotated as "by similarity", "potential", "probable". This resulted to the collection of 66 and 51 sites for O-GlcNAcylated Ser and Thr on 53 proteins. To avoid data redundancy, each data obtained from one database was compared to the data obtained from the other databases based on its O-GlcNAcylated site position and the UniProtKB accession number utilized by all three databases. Redundancy was removed by retaining only one record in the event of finding multiple records of the same site position and accession number. After the removal of redundant data, we have obtained 261 and 149 nonredundant sites for O-GlcNAcylated Ser and Thr on 176 proteins.

As shown in Table 1 the combined non-redundant data of 410 experimentally verified O-GlcNAcylation sites from dbOGAP, OGlycBase and UniProtKB was regarded as the positive data for the investigation of OGT substrate motifs and the construction of predictive models. With an attempt to explore the substrate motifs of O-GlcNAc transferases, sequence fragments were extracted using a window length of 11 centered on O-GlcNAcylated Ser and Thr residues $[33,34]$. In this investigation, the sequence fragments centered on non-O-GlcNAcylated Ser and Thr residues were regarded as negative training data. After removing identical sequence fragments, a total of 17381 and 10587 negative sequence fragments for Ser and Thr residues were obtained from $176 \mathrm{O}$-GlcNAcylated proteins.

\section{Detection of OGT substrate motifs}

The O-GlcNAc transferase (OGT) exhibits substrate site specificity for the sugar donor recognition mechanism and the interaction to target proteins [35]. In this investigation, 
Table 1. Data statistics of positive and negative training data

\begin{tabular}{clccc}
\hline Data resource & Residue & $\begin{array}{c}\text { Number of O-GlcNAcylated } \\
\text { sites (Positive data) }\end{array}$ & $\begin{array}{c}\text { Number of non-O-GlcNAcylated } \\
\text { sites (Negative data) }\end{array}$ & $\begin{array}{c}\text { Number of non-O-GlcNAcylated sites } \\
\text { (Balanced negative data) }\end{array}$ \\
\hline dbOGAP & Serine & 250 & 18,570 & - \\
\cline { 2 - 5 } & Threonine & 142 & 11,240 & - \\
\hline \multirow{2}{*}{ OGlycBase } & Serine & 24 & 1,013 & - \\
\cline { 2 - 5 } & Threonine & 24 & 694 & - \\
\hline \multirow{2}{*}{ UniProtKB } & Serine & 66 & 4,851 & - \\
\cline { 2 - 5 } & Threonine & 51 & 3,255 & 261 \\
\hline \multirow{2}{*}{$\begin{array}{c}\text { Non-redundant } \\
\text { dataset }\end{array}$} & Serine & 261 & 17,381 & 149 \\
\cline { 2 - 5 } & Threonine & 149 & 10,587 & 410 \\
\cline { 2 - 5 } & Combined & 410 & 27,968 & \\
\hline
\end{tabular}

a recursively statistical method, maximal dependence decomposition (MDD) [36], was applied to the positive training data in order to discover substrate motif signatures of O-GlcNAcylation sites by clustering a large-scale dataset of aligned sequences into subgroups that contain statistically significant substrate motifs. MDD extract motifs according to the conserved biochemical property of amino acids. In order to do this, the twenty types of amino acids are categorized into five groups: polar, acidic, basic, hydrophobic, and aromatic groups, as shown in Table S1 (Additional file 1). A contingency table of the amino acids occurrence between two positions is then constructed, as presented in Figure 1. MDD utilizes chi-squared test to test the dependence of amino acid occurrence between two positions, $A_{i}$ and $A_{i}$, surrounding the O-GlcNAcylated site [37]. The chi-squared test implemented in MDD is defined as:

$$
\chi^{2}\left(\mathrm{~A}_{\mathrm{i}}, \mathrm{A}_{\mathrm{j}}\right)=\sum_{m=1}^{5} \sum_{n=1}^{5} \frac{\left(X_{m n}-E_{m n}\right)^{2}}{E_{m n}}
$$

where $X_{m n}$ represented the number of sequences having amino acids from group $m$ in position $A_{i}$ and amino acids from group $n$ in position $A_{j}$, for each pair $\left(A_{i}, A_{j}\right)$ with $i \neq \mathrm{j} . E_{m n}$ is calculated as $\frac{X_{m R} \cdot X_{C n}}{X}$, where $X_{m R}=$ $X_{m 1}+\ldots+X_{m 5}, X_{C n}=X_{1 n}+\ldots+X_{5 n}$, and $X$ denotes the total number of sequences. If a strong dependence is detected (defined as that the chi-square value was larger than 34.3, corresponding to a cutoff level of $P=0.005$ with 16 degrees of freedom) between two positions, then the process is continued as described [38]. Moreover, a minimum cluster size is set when applying MDD to cluster the sequences in the positive training data. If the data size of a subgroup was less than the given parameter, the subgroup will not be divided any further. For this study, MDD was executed using various values in order to obtain an optimal minimum cluster size.

\section{Construction of two-layered prediction model}

In this work, the two-layered machine learning method, incorporating profile hidden Markov model (HMM) and support vector machine (SVM), was used to construct the predictive model from the positive data and negative data of the training set. As presented in Figure 2, profile HMM is generated for each MDD-clustered subgroup in first layer. After applying MDD clustering on O-GlcNAcylated data, the sequence fragments of each MDD-clustered subgroup is taken as a training set to build a profile HMM. An HMM detects distant relationships between amino acid sequences by describing a probability distribution over a potentially infinite number of sequences [39]. In this study, we utilized the software package HMMER [39] in order to build profile HMMs, to calibrate the HMMs, and to search putative O-GlcNAcylation sites against the protein sequences. As models are built based on positive instances of a class, only positive data are utilized to build a predictive model. For each model of the MDD-clustered subgroups, a threshold parameter is selected for identifying potential positive sites from a query [39]. The optimal threshold is the value that gives the most optimal cross-validation performance for each training model. For every search, HMMER returns a bit score and an expectation value (E-value) for each sequence fragment. The bit score is the base two logarithm of the ratio between the probability that the query sequence is a significant match and the probability that the query is produced by a random model. Additionally, the E-value represents the expected number of sequences with a score greater than or equal to the returned HMMER bit scores. A search result with an HMMER bit score greater than the threshold parameter is taken as a positive prediction. While decreasing the bit score threshold favors finding true positives, increasing the bit score threshold favors finding true negatives. Therefore, the threshold must be optimized to obtain a balanced number of true positives and true negatives. 


\section{Construction of aligned O-GIcNAcylated sequences}

A group of aligned

O-GlcNAcylated sequences

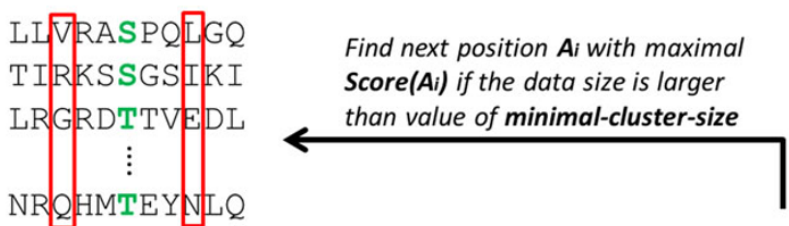

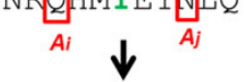

2. A contingency table between two positions $A_{i}$ and $A_{j}$

\begin{tabular}{|l|l|}
\multicolumn{2}{|c|}{ Grouping of amino acids } \\
\hline Group name & Amino acids \\
\hline Polar & G, S, T, C, Q, N \\
\hline Acidic & D, E \\
\hline Basic & K, R, H \\
\hline Hydrophobic & A, V, L, I, P, M \\
\hline Aromatic & F, Y, W \\
\hline
\end{tabular}

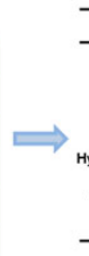

\begin{tabular}{|c|c|c|c|c|c|c|}
\hline$A_{i} \backslash A_{i}$ & Polar & Acidic & Basic & Hydrophobic & Aromatic & Total \\
\hline Polar & $X_{11}$ & $X_{12}$ & $X_{13}$ & $X_{14}$ & $X_{15}$ & $X_{1 R}$ \\
\hline Acidic & $X_{21}$ & $x_{22}$ & $X_{23}$ & $X_{24}$ & $X_{25}$ & $X_{2 R}$ \\
\hline Basic & $X_{31}$ & $X_{32}$ & $X_{33}$ & $X_{34}$ & $X_{35}$ & $X_{3 R}$ \\
\hline Hydrophobic & $X_{41}$ & $X_{42}$ & $X_{43}$ & $X_{44}$ & $X_{65}$ & $X_{A R}$ \\
\hline Aromatic & $X_{51}$ & $X_{52}$ & $X_{53}$ & $X_{54}$ & $X_{55}$ & $X_{S R}$ \\
\hline Total & $X_{C 1}$ & $X_{C 2}$ & $X_{C 3}$ & $X_{C 4}$ & $X_{C 5}$ & $x$ \\
\hline
\end{tabular}

3. Chi-square test

$$
\begin{aligned}
& \chi^{2}\left(A_{i}, A_{j}\right)=\sum_{m=1}^{5} \sum_{n=1}^{5} \frac{\left(X_{m n}-E_{m n}\right)^{2}}{E_{m n}}, E_{m n}=\frac{X_{m R} X_{C n}}{X} \\
& P(\text { null hypothesis is rejected when two positions are dependent }) \\
& =P\left(\chi^{2}\left(A_{i}, A_{j}\right) \geq K \mid \text { null hypothesis }\right)=\alpha
\end{aligned}
$$

\section{Maximal dependence decomposition}

The position Ai with maximal Score $\left(A_{i}\right)$ is applied to cluster sequences according to the amino acid group containing maximal chisquare value.

\section{Calculating the score for each position $A_{i}$} $\operatorname{Score}\left(A_{i}\right)=\sum_{j=-5}^{+5} \chi^{2}\left(A_{i}, A_{j}\right), j \neq i$

Figure 1 Analytical flowchart of MDD clustering

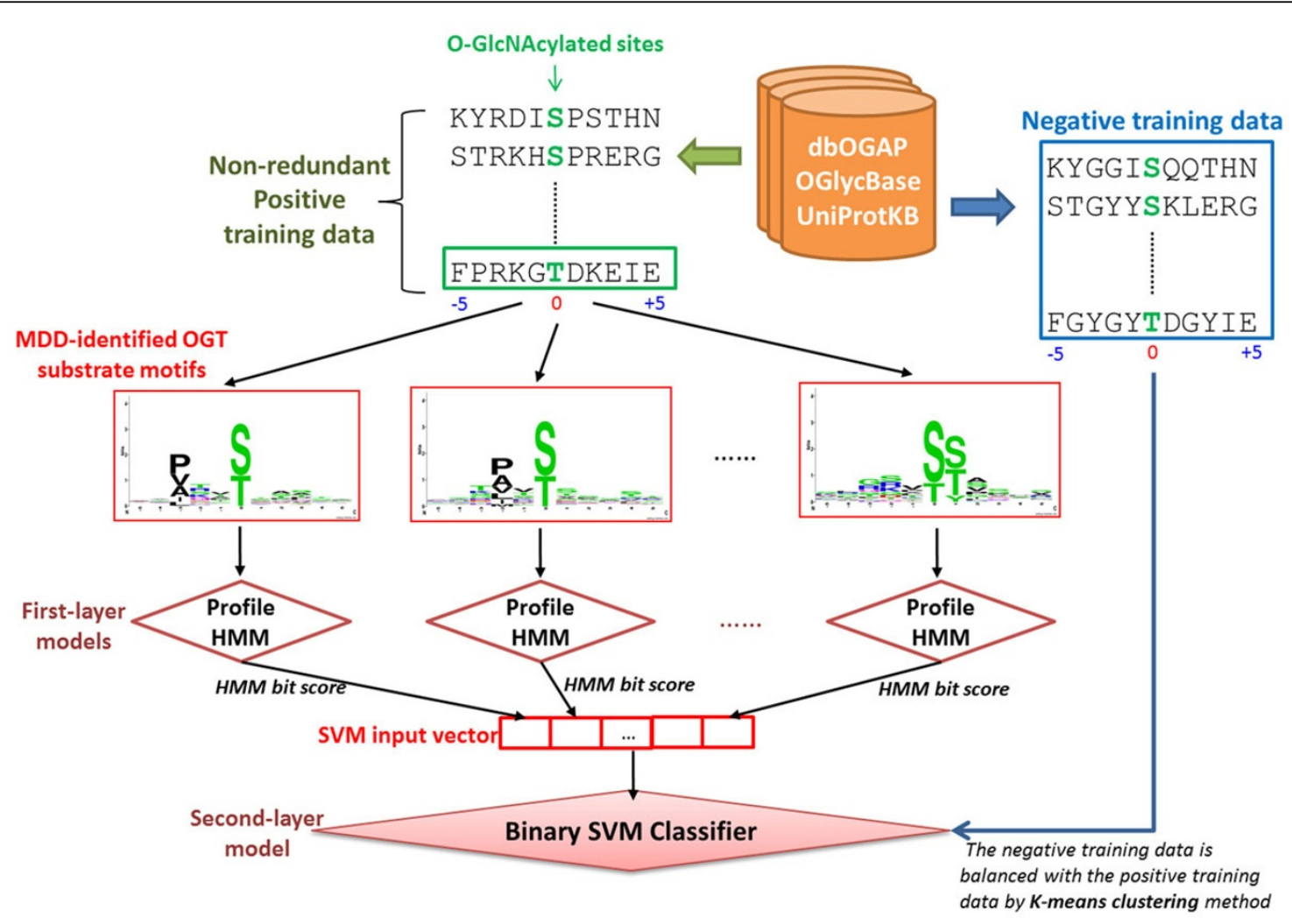

Figure 2 Conceptual diagram of constructing two-layered prediction model from MDD-identified substrate motifs 
In second layer, a binary SVM classifier is trained using the bit scores of profile HMMs. Based on binary classification, SVMs map the input samples into a higher dimensional space using a kernel function. It then finds a hyper-plane that discriminates between the two classes with maximal margin and minimal error. For this study, we employed a public SVM library, LIBSVM [40], to generate the second-layered model from the bit scores of positive and negative training data. The radial basis function $(\mathrm{RBF}) K\left(S_{i}, S_{j}\right)=\exp \left(-\gamma\left\|S_{i}-S_{j}\right\|^{2}\right)$ was used as the kernel function of the SVM. The LIBSVM library is able to produce a probability ranging from 0 to 1 for each prediction; in default, a probability value higher than 0.5 is defined as a positive instance. In order to avoid a biased prediction performance, the negative training data was balanced with the positive training data. To select a representative set of negative data, $K$-means clustering $[36,41]$ was employed with reference to previous PTM prediction methods [42-47]. This resulted in an equal number of positive and negative sequence fragments for the training data (Table 1).

\section{Five-fold cross validation and performance evaluation}

Five-fold cross validation was performed in order to evaluate the predictive performance of each model using various parameters. For this process, the training data is divided into five groups by splitting each dataset into approximately equal sized subgroups where one subgroup is regarded as the test set while the remaining four subgroups are regarded as the training set. This process is repeated five times with each subgroup being used as a test set once [48]. The following measures were used to gauge the average predictive performance of the trained models: Sensitivity $(\mathrm{Sn})=\mathrm{TP} /(\mathrm{TP}+\mathrm{FN})$, Specificity $(\mathrm{Sp})=$ $\mathrm{TN} /(\mathrm{TN}+\mathrm{FP})$, Accuracy $(\mathrm{Acc})=(\mathrm{TP}+\mathrm{TN}) /(\mathrm{TP}+\mathrm{FP}+$ $\mathrm{TN}+\mathrm{FN})$, and Matthews Correlation Coefficient $(\mathrm{MCC})=\frac{(T P \times T N)-(F N \times F P)}{\sqrt{(T P+F N) \times(T N+F P) \times(T P+F P) \times(T N+F N)}}$, where TP, TN, FP and FN represent the numbers of true positives, true negatives, false positives and false negatives, respectively. After thirty rounds of cross-validation process, average Sn, Sp, Acc and MCC values were calculated for each model. The predictive model with the best average performance was then selected for further evaluation by independent testing dataset.

\section{Construction of independent testing data set}

In order to address a potential overestimation of the predictive performance of the models due to over-fitting, an independent test was carried out. For this analysis, experimentally validated sequences obtained from PhosphoSitePlus [49] were used as independent testing data. A total of 779 and 582 experimentally verified sites for O-GlcNAcylated Ser and Thr on 542 proteins were obtained from PhosphoSitePlus. Similar to the construction of positive training set, the sequence fragments centered on O-GlcNAcylated Ser and Thr residues are extracted using 11-mer window length. Additionally, O-GlcNAcylated sequence fragments homologous to the positive training data were removed in order to generate a non-homologous independent testing data. As a result, a total of 956 sequence fragments, consisting of 522 and 434. O-GlcNAcylated Ser and Thr residues, respectively, were regarded as the positive data for independent testing. On the other hand, sequence fragments centered on nonO-GlcNAcylated Ser and Thr residues were regarded as negative data for independent testing. Upon removing homologous data, a total of 60976 sequence fragments (38682 and 22294 non-O-GlcNAcylated Ser and Thr residues) were collected for the negative testing data.

\section{Results and discussion}

Amino acids composition of O-GlcNAcylation sites

This study aims to investigate the OGT substrate motifs based on the amino acid composition surrounding O-GlcNAcylation sites. Figure 3(A) presents the comparison of amino acids composition between positive data (410 O-GlcNAcylated sites) and negative data (27968 non-O-GlcNAcylated sites). O-GlcNAcylated sites appear to conatin more hydrophobic amino acids than non-OGlcNAcylated sites. On the other hand, non-O-GlcNAcylated sites appear to contain more charged amino acids than O-GlcNAcylated sites. Polar amino acids appear to be well distributed in both data sets. The position-specific amino acids composition surrounding the O-GlcNAcylation sites is visualized using WebLogo as shown in Figure 3(B). O-GlcNAcylated Ser/Thr (positive data) residues and unmodified ones (negative data) were centered on position 0 , and the flanking amino acids $(-5 \sim+5)$. The difference between the amino acid composition of $\mathrm{O}$ GlcNAcylated and non-O-GlcNAcylated sites is further visualized using TwoSampleLogo [50], as shown Figure 3 (C). It can be clearly observed that the most pronounced feature of O-GlcNAcylation sites is the abundance of hydrophobic amino acids Proline (P), Valine (V), and Alanine (A), locating centrally around position -2 and +3 . Besides, the polar amino acids, Threonine $(\mathrm{T})$ and Serine (S), also located centrally at position -1 and +1 . Additionally, charged amino acids, especially the positively charged Lysine (K) and Arginine (R) were dominant at position $-2,-4$ and -5 , suggesting that the distant amino acids in sequence, which may be close to O-GlcNAcylation sites in three-dimensional structure, showed notable difference between modified and unmodified sites. Another featured characteristic is the depletion of $P$ and $\mathrm{L}$ at +1 and +2 , respectively, which is immediately adjacent to the O-GlcNAcylation sites. It should also be 


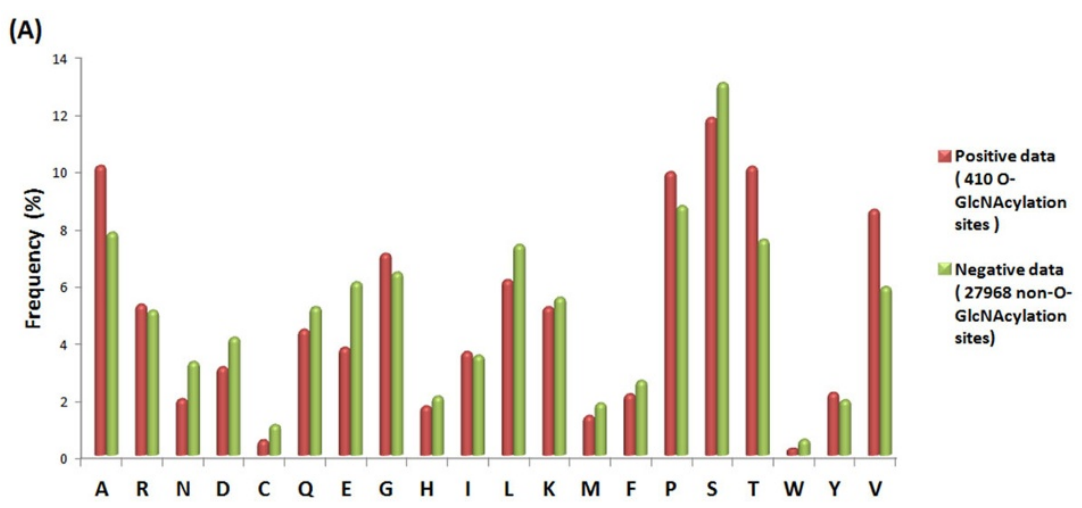

(B)

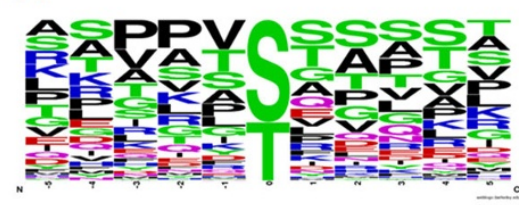

(C)

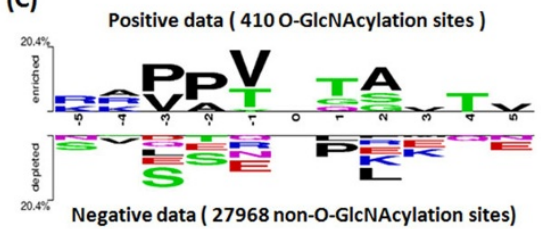

Figure 3 Amino acids composition surrounding the O-GlcNAcylation sites. (A) Comparison of amino acids composition between positive data (410 O-GlcNAcylation sites) and negative data (27968 non-O-GlcNAcylation sites). (B) Position-specific amino acids composition surrounding the O-GlcNAcylation sites. (C) TwoSampleLogo $(p$-value<0.05) between positive data and negative data.

noted that $\mathrm{S}, \mathrm{T}$, and Glutamate (E) were also found to be less frequent around position $-2,-3$, and +5 .

\section{Substrate site motifs of O-GIcNAc transferases}

This study focuses on the characterization of potential OGT substrate motifs based on the amino acid composition surrounding O-GlcNAcylation sites. In order to detect the potential OGT substrate motifs from large scale O-GlcNAcylation data set, we applied the MDD to further cluster all 410 experimentally verified $\mathrm{O}$ GlcNAcylated peptide sequences into subgroups by iteratively capturing the positions with maximal dependence of amino acids composition. As illustrated in Figure 4, the MDD-identified substrate motifs were visualized in a tree-like structure. Firstly, position -3 had the maximal dependence with the occurrence of hydrophobic amino acid group. Subsequently, all 205 sites containing the hydrophobic group in position -3 could be further divided into two subgroups: subgroup OGT1 (100 sites) represented the occurrence of hydrophobic amino acids in position - 2 with maximal dependence, whereas subgroup OGT2 (105 sites) had no occurrence of hydrophobic amino acids in position -2 . It would be noticed that the subgroup OGT1 gives the substrate motif of hydrophobic amino acids in both positions -3 and -2 , which is consistent with the consensus motif previously suggested as P-P-T-[ST]-T-A [22]. In right subtree, the data (205 sites) without the hydrophobic amino acids in position -3 were divided into two subgroups: subgroup OGT3 (95 sites) involved the maximal dependence of hydrophobic amino acids in position -2, yet the other (110 sites) had no occurrence of hydrophobic amino acids in position -2. Furthermore, the 110 sites containing could be divided into two subgroups: subgroup OGT4 (39 sites) represented the occurrence of hydrophobic amino acids in position -1 while the other (71 sites) had no occurrence of hydrophobic amino acids in position - 1 . The hydrophobic residues indicate its contribution in the interfaces of protein-protein interactions. Finally, totally seven OGT substrate motifs (marked in red) were identified with significant dependences $(P$-value $<0.005)$. Subgroups OGT5 and OGT6 depicted the conserved motifs of polar amino acids at positions +1 and -1 , respectively. However, subgroup OGT7, that contains the remaining 22 O-GlcNAcylation sites, had a little conserved motif of glycine $(\mathrm{G})$ residue at position -1. Interestingly, the small size and flexibility of $G$ residue is probably responsible for making it suitable for the structural adjustments required during the protein-protein interactions [51]. Table S2 (Additional file 2) shows the number of O-GlcNAcylation sites (positive data) in each MDD-identified OGT substrate motif.

Predictive performance of the identified substrate motifs To identify how to best classify O-GlcNAcylation from non-O-GlcNAcylation sites, the predictive models were trained with each of the following: OGT1, OGT2, 


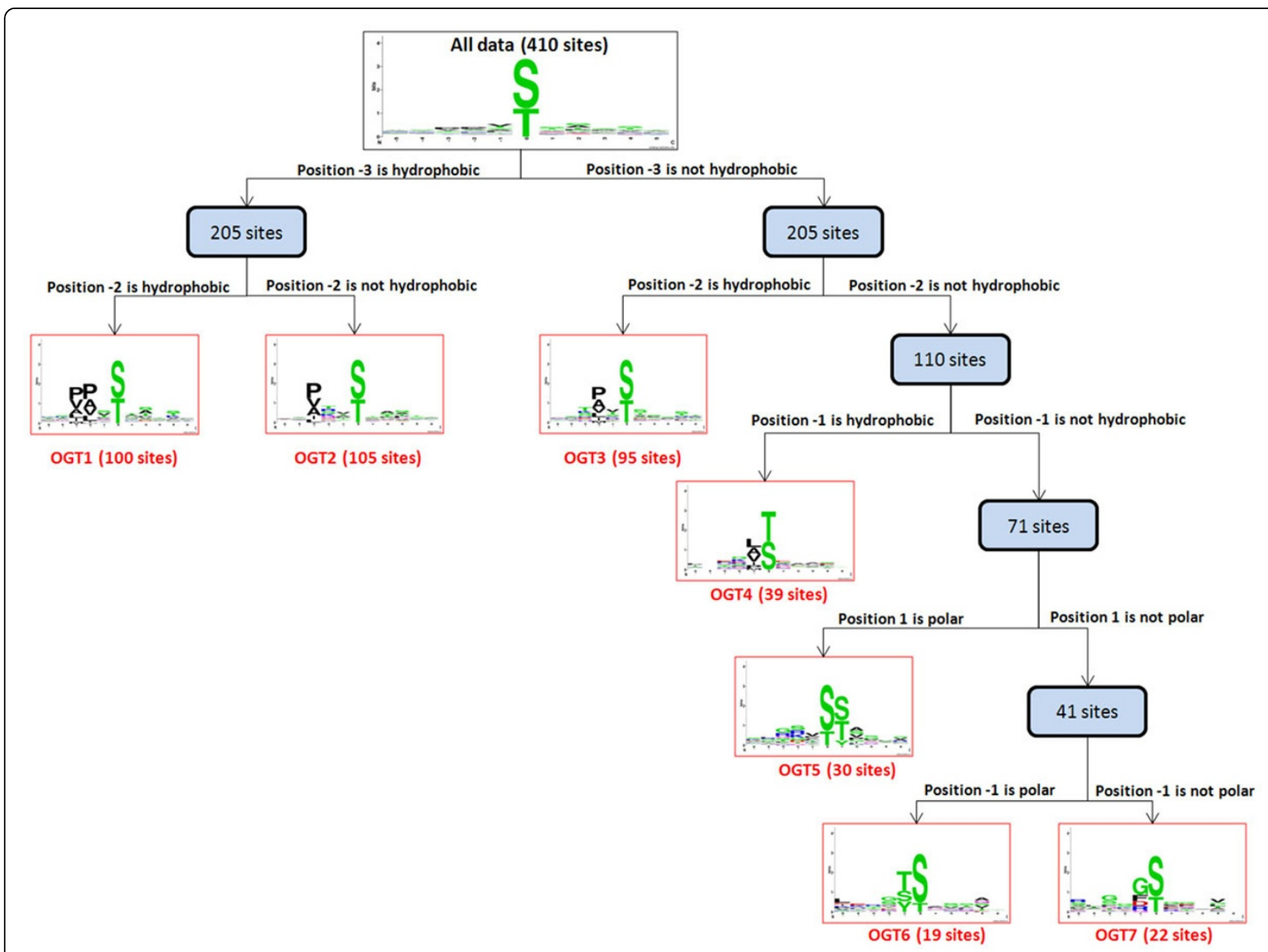

Figure 4 The tree view of potential OGT substrate motifs identified by MDD clustering on 410 O-GlcNAcylation sites

OGT3, OGT4, OGT5, OGT6, OGT7, as well as all OGTs combined. The predictive power of each model was evaluated by measuring the sensitivity $(\mathrm{Sn})$, specificity $(\mathrm{Sp})$, accuracy (Acc), and Matthews correlation coefficient (MCC). According to evaluation of five-fold cross-validation, the single HMM trained from all 410 positive data yield a sensitivity of $68.8 \%$, a specificity of $70.7 \%$, an accuracy of $69.8 \%$, and an MCC value of 0.395 . As shown in Table 2 the HMM trained from OGT1 subgroup, that contains a conserved motif of hydrophobic amino acids at positions -2 and -3 , provided the highest predictive power with a sensitivity of $93.0 \%$, a specificity of $89.0 \%$, an accuracy of $91.0 \%$, and an MCC value of 0.821 . Among all others, the HMM trained from OGT4 subgroup yielded the lowest sensitivity $0.71 .8 \%$, while the HMM trained from OGT7 subgroup yielded the lowest specificity at $68.2 \%$. Among the seven subgroups, the HMM trained from OGT7 subgroup provided a lowest accuracy at $70.5 \%$. Additionally, combining seven OGT HMMs (MDD-clustered HMMs) could achieve the predictive performance of $83.7 \%$ sensitivity, $77.1 \%$ specificity, $80.4 \%$ accuracy, and 0.609 MCC value. This investigation indicated that the application of MDD clustering could improve the performance on the prediction of protein O-GlcNAcylation sites.

With reference to a previous work applying two-layered SVMs on the prediction of viral phosphorylation sites [52], this work further combined seven profile HMMs (first layer) and one SVM (second layer) into a two-layered prediction model, which provides a better performance than the combination of seven OGT HMMs (MDD-clustered HMMs). The two-layered prediction model yielded a sensitivity of $85.4 \%$, a specificity of $84.1 \%$, an accuracy of $0.84 .7 \%$, and an MCC value of 0.695 . In this investigation, the model providing best performance was further evaluated by independent testing set.

\section{Independent testing and comparison with other prediction tools}

The final non-redundant data of independent testing set consisting of 956 positive sites and 60976 negative sites was utilized for further evaluating the constructed models. As shown in Table 3 the single HMM trained using 
Table 2. Five-fold cross validation results on profile HMMs learned from all data and seven MDD-clustered subgroups

\begin{tabular}{|c|c|c|c|c|c|c|}
\hline Models & Number of positive data & Number of negative data & Sn & Sp & Acc & MCC \\
\hline Single HMM with all data & 410 & 410 & $68.8 \%$ & $70.7 \%$ & $69.8 \%$ & 0.395 \\
\hline HMM with OGT1 & 100 & 100 & $93.0 \%$ & $89.0 \%$ & $91.0 \%$ & 0.821 \\
\hline HMM with OGT2 & 105 & 105 & $83.8 \%$ & $71.4 \%$ & $77.6 \%$ & 0.557 \\
\hline HMM with OGT3 & 95 & 95 & $85.3 \%$ & $75.8 \%$ & $80.5 \%$ & 0.613 \\
\hline HMM with OGT4 & 39 & 39 & $71.8 \%$ & $74.4 \%$ & $73.1 \%$ & 0.462 \\
\hline HMM with OGT5 & 30 & 30 & $73.3 \%$ & $73.3 \%$ & $73.3 \%$ & 0.467 \\
\hline HMM with OGT6 & 19 & 19 & $78.9 \%$ & $73.7 \%$ & $76.3 \%$ & 0.527 \\
\hline HMM with OGT7 & 22 & 22 & $72.7 \%$ & $68.2 \%$ & $70.5 \%$ & 0.410 \\
\hline MDD-clustered HMMs (Combined 7 OGT HMMs) & 410 & 410 & $83.7 \%$ & $77.1 \%$ & $80.4 \%$ & 0.609 \\
\hline Two-layered model (7 HMMs + 1 SVM) & 410 & 410 & $85.4 \%$ & $84.1 \%$ & $84.7 \%$ & 0.695 \\
\hline
\end{tabular}

Table 3. The comparison of independent testing results between our methods and other three O-GIcNAcylation prediction tools

\begin{tabular}{|c|c|c|c|c|c|c|c|c|}
\hline Methods & TP & FN & TN & FP & Sn & $\mathrm{Sp}$ & Acc & MCC \\
\hline Single HMM with all data & 609 & 347 & 40072 & 20904 & $63.70 \%$ & $65.72 \%$ & $65.69 \%$ & 0.076 \\
\hline $\begin{array}{l}\text { MDD-clustered HMMs } \\
\text { (7 OGT HMMs) }\end{array}$ & 833 & 123 & 45212 & 15764 & $87.13 \%$ & $74.15 \%$ & $74.38 \%$ & 0.171 \\
\hline $\begin{array}{l}\text { Two-layered model } \\
\text { (7 HMMs + } 1 \text { SVM) }\end{array}$ & 828 & 128 & 51224 & 9752 & $86.61 \%$ & $84.01 \%$ & $84.05 \%$ & 0.231 \\
\hline YinOYang & 449 & 507 & 50619 & 10357 & $46.97 \%$ & $83.01 \%$ & $82.46 \%$ & 0.097 \\
\hline O-GlcNAcScan & 411 & 545 & 51219 & 9757 & $42.99 \%$ & $84.00 \%$ & $83.37 \%$ & 0.089 \\
\hline O-GICNACPRED & 554 & 402 & 38414 & 22562 & $57.95 \%$ & $63.00 \%$ & $62.92 \%$ & 0.053 \\
\hline
\end{tabular}

all positive data achieved a sensitivity of $63.70 \%$, a specificity of $65.72 \%$, an accuracy of $65.69 \%$, and an $\mathrm{MCC}$ value of 0.076 . The MDD-clustered HMMs achieved a sensitivity of $87.13 \%$, a specificity of $74.15 \%$, an accuracy of $74.38 \%$, and an MCC value of 0.171 . This investigation indicated that a greater prediction power could be obtained by using MDD-clustered HMM models than that by a single HMM model. Additionally, the twolayered model achieved a sensitivity of $86.61 \%$, a specificity of $84.01 \%$, an accuracy of $84.05 \%$, and an MCC value of 0.231 . The independent testing demonstrated that the two-layered model could perform better than MDDclustered HMMs and could provide a promising accuracy for 542 experimentally verified O-GlcNAcylated proteins, which were not considered within the construction of predictive model.

To further demonstrate the effectiveness of our method, the independent testing set was used to compare the twolayered model with three popular O-GlcNAcylation site prediction tools, YinOYang, O-GlcNAcScan, and O-GlcNAcPRED. Table 3 indicated that the prediction power yielded by our two-layered model was superior to that by other three prediction tools. By using default threshold value (0.5), YinOYang yielded a sensitivity of $46.97 \%$, a specificity of $83.01 \%$, an accuracy of $82.46 \%$, and an MCC value of 0.097 . O-GlcNAcScan achieved a sensitivity of $42.99 \%$, a specificity of $84.00 \%$, an accuracy of $83.37 \%$, and an MCC value of 0.089 . O-GlcNAcPRED provided a lowest independent testing performance: $57.95 \%$ sensitivity, $63.00 \%$ specificity, $62.92 \%$ accuracy, and $0.053 \mathrm{MCC}$ value. This independent testing indicated that the two-layered model could provide balanced sensitivity and specificity for such unbalanced positive and negative datasets. The proposed method also provided comparable accuracy with that analyzed by O-GlcNAcScan. Overall, as presented in Figure S1 (Additional file 3), the proposed method outperformed the three prediction tools.

\section{Web-based system for the identification of 0 -} GIcNAcylation sites

With the time-consuming and lab-intensive experimental identification of protein O-GlcNAcylation sites, a biologist may only concluded that a protein can be O-GlcNAcylated but the precise O-GlcNAcylation sites remains unknown. Therefore, an effective prediction server can help to focus efficiently on potential sites. After evaluation by crossvalidation and independent testing, the two-layered model with best predictive performance was adopted to implement a web-based system, named OGTSite, for predicting O-GlcNAcylated sites with potential OGT substrate motifs. As shown in Figure 5, users can submit their protein sequences in FASTA format or just provide the UniProtKB 


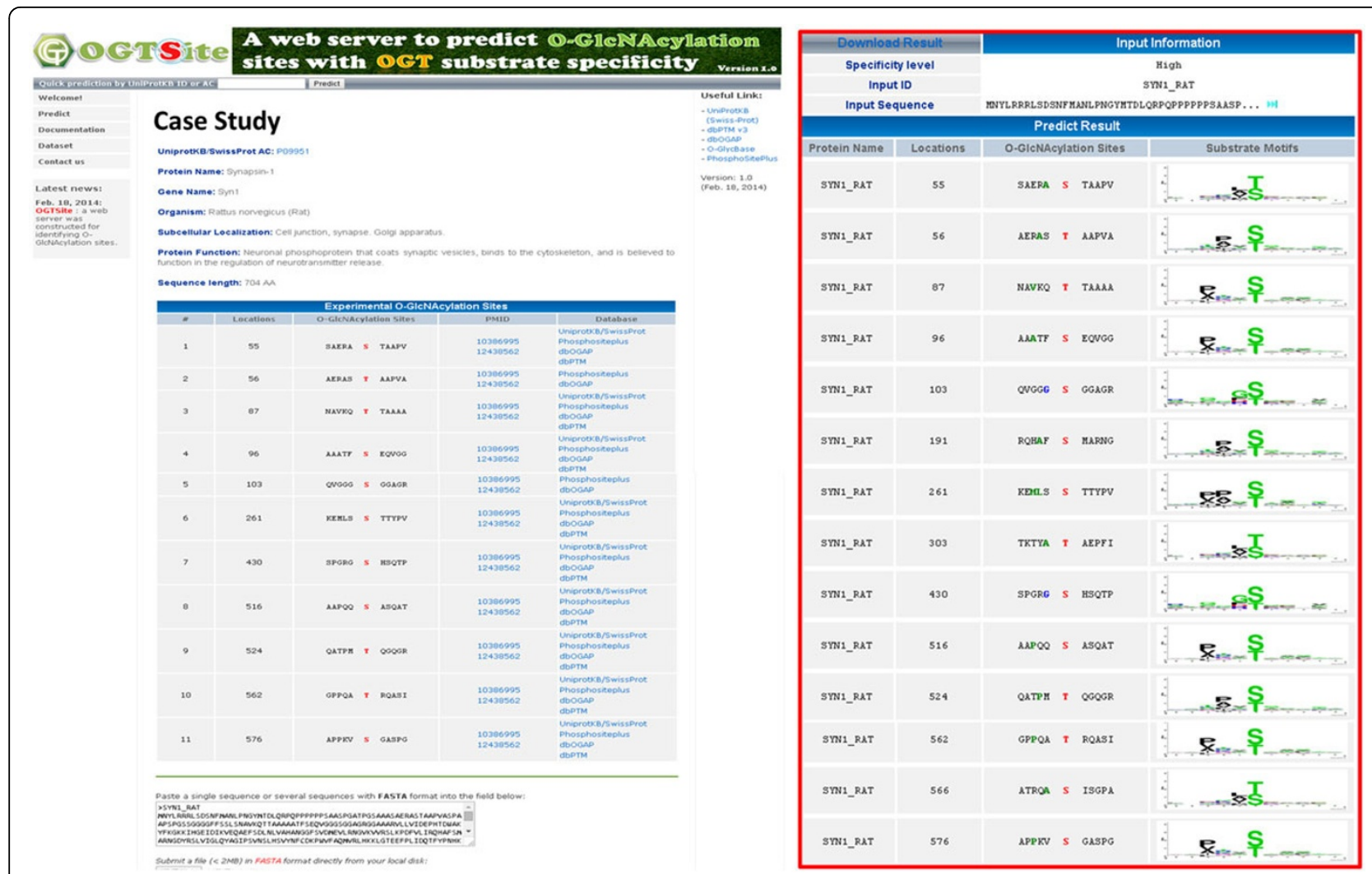

Figure 5 A case study of O-GIcNAcylation sites prediction on Synapsin-1 (Syn1) of Rattus norvegicus.

accession number. The system returns the predictions, including O-GlcNAcylated position and flanking amino acids. Users can also access the substrate motifs used for predicting the O-GlcNAcylation sites. Take Synapsin-1 (Syn1) of Rattus norvegicus as an example, 11 sites such as S55, T56, T87, S96, S103, S261, S430, S516, T524, T562 and S576 have been experimentally verified as O-GlcNAcylation sites [53]. OGTSite predicted a total of 14 potential O-GlcNAcylation sites, including 11 true positive predictions. Although S191, T303 and T566 have not yet been validated as the O-GlcNAcylation sites, they have the potential OGT3 and OGT4 substrate motifs, respectively. This case study suggests the feasibility of this model to identify the S/T residues that can be modified by O-GlcNAc moiety.

\section{Conclusion}

This study presents a novel scheme to identify potential substrate specificity of O-GlcNAc transferase based on a set of experimentally verified O-GlcNAcylation sites. We have demonstrated the utility of MDD clustering method in the characterization of substrate motifs of O-GlcNAcylation sites. Additionally, the proposed pipeline includes the effectiveness of the identified MDDdetected short linear motifs to predict O-GlcNAcylated sites. A five-fold cross-validation evaluation showed the power of MDD-identified substrate motifs in the prediction of O-GlcNAcylated sites. Moreover, the two-layered model combining seven profile HMMs and one SVM could provide the best performance. The two-layered model has been used to implement an online system, OGTSite, for an effective identification of protein O-GlcNAcylation sites. By identifying potential O-GlcNAcylation sites using the proposed method, we will be providing a reliable lead to the scientific community to minimize costs and effort for experimentally verifying actual O-GlcNAcylation sites. It should be noted that the proposed method could also be extended to include more meaningful substrate motifs by further acquiring experimentally verified O-GlcNAcylation sites. Additionally, a more abundant set of experimentally verified O-GlcNAcylation sites with protein tertiary structure information could be used to strengthen site prediction capabilities [54].

\section{Availability}

The proposed method is implemented as a web-based resource, which is now freely available to all interested users at http://csb.cse.yzu.edu.tw/OGTSite/. All of the dataset used in this work is also available for download in the website. 


\section{Additional material}

Additional file 1: Table S1. The grouping of twenty amino acids used in this study.

Additional file 2: Table S2. The identified OGT substrate motifs of 410 O-GlcNAcylation sites

Additional file 3: Figure S1. The comparison of independent testing results between our methods and other three O-GlcNAcylation prediction tools.

\section{Competing interests}

The authors declare that they have no competing interests.

\section{Authors' contributions}

TYL and SLW conceived and supervised the project. HJK, CHH, CTL and KYH were responsible for the design, computational analyses, implemented the web-based tool, and drafted the manuscript with revisions provided by NAB and TYL. All authors read and approved the final manuscript.

\section{Acknowledgements}

The authors sincerely appreciate the Ministry of Science and Technology (MOST) of Taiwan for financially supporting this research under contract number of MOST 103-2221-E-155-020-MY3, MOST 103-2633-E-155-002, and MOST 104-2221-E-155-036-MY2.

\section{Declarations}

Publication charge for this work was funded by MOST grant under contract number of MOST 103-2221-E-155-020-MY3 and MOST 104-2221-E-155-036MY2 to TYL.

This article has been published as part of BMC Bioinformatics Volume 16 Supplement 18, 2015: Joint 26th Genome Informatics Workshop and 14th International Conference on Bioinformatics: Bioinformatics. The full contents of the supplement are available online at http://www.biomedcentral.com/ bmcbioinformatics/supplements/16/S18.

\section{Authors' details}

${ }^{1}$ Department of Computer Science and Engineering, Yuan Ze University, Taoyuan 320, Taiwan. ${ }^{2}$ Tao-Yuan Hospital, Ministry of Health \& Welfare, Taoyuan 320, Taiwan. ${ }^{3}$ Inflammation and Infection Research Centre, School of Medical Sciences, University of New South Wales, Sydney, Australia. ${ }^{4}$ Department of Obstetrics and Gynecology, Hsinchu Mackay Memorial Hospital, Hsin-Chu 300, Taiwan. ${ }^{5}$ Mackay Junior College of Medicine, Nursing and Management, Taipei 112, Taiwan. ${ }^{6}$ Department of Medicine, Mackay Medical College, New Taipei City 252, Taiwan. ${ }^{7}$ Innovation Center for Big Data and Digital Convergence, Yuan Ze University, Taoyuan 320, Taiwan.

Published: 9 December 2015

\section{References}

1. Hart GW, Housley MP, Slawson C: Cycling of O-linked beta-Nacetylglucosamine on nucleocytoplasmic proteins. Nature 2007, 446(7139):1017-1022.

2. Comer Fl, Hart GW: O-GICNAc and the control of gene expression. Biochim Biophys Acta 1999, 1473(1):161-171.

3. Ogawa M, Furukawa K, Okajima T: Extracellular O-linked beta-Nacetylglucosamine: Its biology and relationship to human disease. World J Biol Chem 2014, 5(2):224-230.

4. Sakaidani Y, Nomura T, Matsuura A, Ito M, Suzuki E, Murakami K, Nadano D, Matsuda T, Furukawa K, Okajima T: O-linked-N-acetylglucosamine on extracellular protein domains mediates epithelial cell-matrix interactions. Nat Commun 2011, 2:583.

5. Delporte A, De Zaeytijd J, De Storme N, Azmi A, Geelen D, Smagghe G, Guisez Y, Van Damme EJ: Cell cycle-dependent O-GlcNAc modification of tobacco histones and their interaction with the tobacco lectin. Plant Physiol Biochem 2014, 83:151-158.

6. Ferrer $C M$, Reginato $M J$ : Cancer metabolism: cross talk between signaling and O-GlcNAcylation. Methods Mol Biol 2014, 1176:73-88.
7. Jozwiak P, Forma E, Brys M, Krzeslak A: O-GIcNAcylation and Metabolic Reprograming in Cancer. Front Endocrinol (Lausanne) 2014, 5:145.

8. McClain DA, Crook ED: Hexosamines and insulin resistance. Diabetes 1996, 45(8):1003-1009

9. Liu F, labal K, Grundke-labal I, Hart GW, Gong CX: O-GlcNAcylation regulates phosphorylation of tau: a mechanism involved in Alzheimer's disease. Proc Natl Acad Sci USA 2004, 101(29):10804-10809.

10. Mi W, Gu Y, Han C, Liu H, Fan Q, Zhang X, Cong Q, Yu W: O-GlcNAcylation is a novel regulator of lung and colon cancer malignancy. Biochim Biophys Acta 2011, 1812(4):514-519.

11. Fardini $Y$, Dehennaut V, Lefebvre T, Issad T: O-GlcNAcylation: A New Cancer Hallmark? Front Endocrinol (Lausanne) 2013, 4:99.

12. Huang $X$, Pan $Q$, Sun D, Chen W, Shen A, Huang M, Ding J, Geng M: O-GIcNAcylation of cofilin promotes breast cancer cell invasion. J Biol Chem 2013, 288(51):36418-36425.

13. Vosseller K, Trinidad JC, Chalkley RJ, Specht CG, Thalhammer A, Lynn AJ, Snedecor JO, Guan S, Medzihradszky KF, Maltby DA, et al: O-linked Nacetylglucosamine proteomics of postsynaptic density preparations using lectin weak affinity chromatography and mass spectrometry. $\mathrm{Mol}$ Cell Proteomics 2006, 5(5):923-934.

14. Trinidad JC, Barkan DT, Gulledge BF, Thalhammer A, Sali A, Schoepfer R, Burlingame AL: Global identification and characterization of both OGIcNAcylation and phosphorylation at the murine synapse. $\mathrm{Mol} \mathrm{Cell}$ Proteomics 2012, 11(8):215-229.

15. Alfaro JF, Gong CX, Monroe ME, Aldrich JT, Clauss TR, Purvine SO, Wang Z, Camp DG, Shabanowitz J, Stanley P, et al: Tandem mass spectrometry identifies many mouse brain O-GIcNAcylated proteins including EGF domain-specific O-GIcNAc transferase targets. Proc Natl Acad Sci USA 2012, 109(19):7280-7285.

16. Khidekel N, Ficarro SB, Clark PM, Bryan MC, Swaney DL, Rexach JE, Sun YE, Coon JJ, Peters EC, Hsieh-Wilson LC: Probing the dynamics of O-GICNAC glycosylation in the brain using quantitative proteomics. Nat Chem Biol 2007, 3(6):339-348.

17. Myers SA, Panning B, Burlingame AL: Polycomb repressive complex 2 is necessary for the normal site-specific O-GICNAc distribution in mouse embryonic stem cells. Proc Natl Acad Sci USA 2011, 108(23):9490-9495.

18. Nandi A, Sprung R, Barma DK, Zhao Y, Kim SC, Falck JR: Global identification of O-GICNAc-modified proteins. Anal Chem 2006, 78(2):452-458.

19. Wang Z, Udeshi ND, O'Malley M, Shabanowitz J, Hunt DF, Hart GW: Enrichment and site mapping of O-linked $\mathrm{N}$-acetylglucosamine by a combination of chemical/enzymatic tagging, photochemical cleavage, and electron transfer dissociation mass spectrometry. Mol Cell Proteomics 2010, 9(1):153-160.

20. Gupta R, Brunak S: Prediction of glycosylation across the human proteome and the correlation to protein function. Pac Symp Biocomput 2002, 310-322.

21. Chen SA, Lee TY, Ou YY: Incorporating significant amino acid pairs to identify O-linked glycosylation sites on transmembrane proteins and non-transmembrane proteins. BMC Bioinformatics 2010, 11:536.

22. Wang J, Torii M, Liu H, Hart GW, Hu ZZ: dbOGAP - an integrated bioinformatics resource for protein O-GICNAcylation. BMC Bioinformatics 2011, 12:91

23. Jia CZ, Liu T, Wang ZP: O-GIcNAcPRED: a sensitive predictor to capture protein O-GICNAcylation sites. Mol Biosyst 2013, 9(11):2909-2913.

24. Caragea C, Sinapov J, Silvescu A, Dobbs D, Honavar V: Glycosylation site prediction using ensembles of Support Vector Machine classifiers. BMC Bioinformatics 2007, 8:438.

25. Vocadlo DJ: O-GlcNAc processing enzymes: catalytic mechanisms, substrate specificity, and enzyme regulation. Curr Opin Chem Biol 2012, 16(5-6):488-497.

26. Wu HY, Lu CT, Kao HJ, Chen YJ, Chen YJ, Lee TY: Characterization and identification of protein O-GICNAcylation sites with substrate specificity. BMC bioinformatics 2014, 15(Suppl 16):S1.

27. Wuhrer M, Catalina MI, Deelder AM, Hokke CH: Glycoproteomics based on tandem mass spectrometry of glycopeptides. J Chromatogr B Analyt Technol Biomed Life Sci 2007, 849(1-2):115-128.

28. Lee TY, Huang HD, Hung JH, Huang HY, Yang YS, Wang TH: dbPTM: an information repository of protein post-translational modification. Nucleic Acids Res 2006, 34(Database):D622-627. 
29. Lu CT, Huang KY, Su MG, Lee TY, Bretana NA, Chang WC, Chen YJ, Huang HD: DbPTM 3.0: an informative resource for investigating substrate site specificity and functional association of protein posttranslational modifications. Nucleic Acids Res 2013, 41(Database):D295-305.

30. Su MG, Huang KY, Lu CT, Kao HJ, Chang YH, Lee TY: topPTM: a new module of dbPTM for identifying functional post-translational modifications in transmembrane proteins. Nucleic Acids Res 2014 42(Database):D537-545.

31. Gupta R, Birch H, Rapacki K, Brunak S, Hansen JE: O-GLYCBASE version 4.0: a revised database of O-glycosylated proteins. Nucleic Acids Res 1999, 27(1):370-372.

32. Apweiler R, Bairoch A, Wu CH, Barker WC, Boeckmann B, Ferro S, Gasteiger E, Huang H, Lopez R, Magrane M, et al: UniProt: the Universal Protein knowledgebase. Nucleic Acids Res 2004, 32(Database):D115-119.

33. Huang HD, Lee TY, Tzeng SW, Wu LC, Horng JT, Tsou AP, Huang KT: Incorporating hidden Markov models for identifying protein kinasespecific phosphorylation sites. J Comput Chem 2005, 26(10):1032-1041.

34. Huang HD, Lee TY, Tzeng SW, Horng JT: KinasePhos: a web tool for identifying protein kinase-specific phosphorylation sites. Nucleic Acids Res 2005, 33(Web Server):W226-229.

35. Ma X, Liu P, Yan H, Sun H, Liu X, Zhou F, Li L, Chen Y, Muthana MM, Chen $X$, et al: Substrate specificity provides insights into the sugar donor recognition mechanism of O-GlcNAc transferase (OGT). PLoS One 2013, 8(5):e63452.

36. Lee TY, Lin ZQ, Hsieh SJ, Bretana NA, Lu CT: Exploiting maximal dependence decomposition to identify conserved motifs from a group of aligned signal sequences. Bioinformatics 2011, 27(13):1780-1787.

37. Nguyen VN, Huang KY, Huang CH, Chang TH, Bretana N, Lai K, Weng J, Lee TY: Characterization and identification of ubiquitin conjugation sites with E3 ligase recognition specificities. BMC bioinformatics 2015, 16(Suppl 1):S1.

38. Burge C, Karlin S: Prediction of complete gene structures in human genomic DNA. J Mol Biol 1997, 268(1):78-94

39. Eddy SR: Profile hidden Markov models. Bioinformatics 1998, 14(9):755-763.

40. Chang CC, Lin CJ: LIBSVM : a library for support vector machines. ACM Transactions on Intelligent Systems and Technology 2011, 2(27):1-27.

41. Shien DM, Lee TY, Chang WC, Hsu JB, Horng JT, Hsu PC, Wang TY, Huang HD: Incorporating structural characteristics for identification of protein methylation sites. J Comput Chem 2009, 30(9):1532-1543.

42. Lee TY, Bretana NA, Lu CT: PlantPhos: using maximal dependence decomposition to identify plant phosphorylation sites with substrate site specificity. BMC Bioinformatics 2011, 12:261

43. Lee TY, Bo-Kai Hsu J, Chang WC, Huang HD: RegPhos: a system to explore the protein kinase-substrate phosphorylation network in humans. Nucleic Acids Res 2011, 39(Database):D777-787.

44. Xue Y, Ren J, Gao X, Jin C, Wen L, Yao X: GPS 2.0, a tool to predict kinasespecific phosphorylation sites in hierarchy. Mol Cell Proteomics 2008, 7(9):1598-1608.

45. Wong $\mathrm{YH}$, Lee TY, Liang HK, Huang $\mathrm{CM}$, Wang TY, Yang $\mathrm{YH}$, Chu $\mathrm{CH}$, Huang HD, Ko MT, Hwang JK: KinasePhos 2.0: a web server for identifying protein kinase-specific phosphorylation sites based on sequences and coupling patterns. Nucleic Acids Res 2007, 35(Web Server):W588-594.

46. Xue Y, Li A, Wang L, Feng H, Yao X: PPSP: prediction of PK-specific phosphorylation site with Bayesian decision theory. BMC Bioinformatics 2006, 7:163

47. Huang KY, Wu HY, Chen YJ, Lu CT, Su MG, Hsieh YC, Tsai CM, Lin Kl, Huang HD, Lee TY, et al: RegPhos 2.0: an updated resource to explore protein kinase-substrate phosphorylation networks in mammals. Database: the journal of biological databases and curation 2014, 2014 bau034

48. Lu CT, Chen SA, Bretana NA, Cheng TH, Lee TY: Carboxylator: incorporating solvent-accessible surface area for identifying protein carboxylation sites. J Comput Aided Mol Des 2011, 25(10):987-995.

49. Hornbeck PV, Kornhauser JM, Tkachev S, Zhang B, Skrzypek E, Murray B, Latham V, Sullivan M: PhosphoSitePlus: a comprehensive resource for investigating the structure and function of experimentally determined post-translational modifications in man and mouse. Nucleic Acids Res 2012, 40(Database):D261-270.

50. Vacic V, lakoucheva LM, Radivojac P: Two Sample Logo: a graphical representation of the differences between two sets of sequence alignments. Bioinformatics 2006, 22(12):1536-1537.
51. Kumar M, Gromiha MM, Raghava GP: Prediction of RNA binding sites in a protein using SVM and PSSM profile. Proteins 2008, 71(1):189-194.

52. Huang KY, Lu CT, Bretana N, Lee TY, Chang TH: ViralPhos: incorporating a recursively statistical method to predict phosphorylation sites on virus proteins. BMC Bioinformatics 2013, 14(Suppl 16):S10.

53. Dias WB, Cheung WD, Wang Z, Hart GW: Regulation of calcium/ calmodulin-dependent kinase IV by O-GlcNAc modification. J Biol Chem 2009, 284(32):21327-21337.

54. Su MG, Lee TY: Incorporating substrate sequence motifs and spatial amino acid composition to identify kinase-specific phosphorylation sites on protein three-dimensional structures. BMC Bioinformatics 2013, 14(Suppl 16):S2

doi:10.1186/1471-2105-16-S18-S10

Cite this article as: Kao et al:: A two-layered machine learning method to identify protein O-GIcNAcylation sites with O-GIcNAc transferase substrate motifs. BMC Bioinformatics 2015 16(Suppl 18):S10.

\section{Submit your next manuscript to BioMed Central and take full advantage of:}

- Convenient online submission

- Thorough peer review

- No space constraints or color figure charges

- Immediate publication on acceptance

- Inclusion in PubMed, CAS, Scopus and Google Scholar

- Research which is freely available for redistribution
C Biomed Central 